IFFC preprint 96-01

\title{
Extended knots and the space of states of quantum gravity* $^{*}$
}

\author{
Jorge Griego \\ Instituto de Física, Facultad de Ciencias, \\ Tristán Narvaja 1674, 11200 Montevideo, Uruguay. \\ E-mail: griego@fisica.edu.uy
}

January 6, 1996

\begin{abstract}
In the loop representation the quantum constraints of gravity can be solved. This fact allowed significant progress in the understanding of the space of states of the theory. The analysis of the constraints over loop dependent wavefunctions has been traditionally based upon geometric (in contrast to analytic) properties of the loops. The reason for this preferred way is twofold: for one hand the inherent difficulties associated with the analytic loop calculus, and on the other our limited knowledge about the analytic properties of knots invariants. Extended loops provide a way to overcome the difficulties at both levels. For one hand, a systematic method to construct analytic expressions of diffeomorphism invariants (the extended knots) in terms of the Chern-Simons propagators can be developed. Extended knots are simply related to ordinary knots (at least formally). The analytic expressions of knot invariants could be produced then in a generic way. On the other hand, the evaluation of the Hamiltonian over extended loop wavefunctions can be thoroughly accomplished in the extended loop framework. These two ingredients promote extended loops as a potential resort for answering important questions about quantum gravity.
\end{abstract}

\section{Introduction}

Knot theory and quantum gravity have a profound relationship that is put into manifest within the framework of the Ashtekar new variable reformulation

*submitted to Nucl. Phys. B 
of general relativity [1]. The facts that i) a loop representation of the Liealgebra valued Ashtekar connection can be introduced [2]; ii) the quantum states of gravity are given in terms of knot invariants due to the diffeomorphism invariance of the theory; iii) there exist knot invariants that are solutions of the Wheeler-DeWitt equation [2, 3]; clearly show the capability of knots to capture relevant information about the quantum properties of space-time.

The relationship between knots and physics goes far beyond the interest of general relativity [4]. In fact, the many ways that knot theory interacts with physics has motivated a renewed interest of physicists to adventure into the traditionally mathematician world of knots (the first goal was in the physics of the late 1800's with the theory of vortex atoms of Lord Kelvin). Part of the recent work was devoted to study in depth the significant relationship between topological quantum field theories and knot polynomials [5, 6, 7], and in particular for the quantum gravity case the specific relation between some knot polynomials and the solutions of the Hamiltonian constraint in the loop representation [8].

In gravitation, until the appearance of the results of Brügmann, Gambini and Pullin [9] the calculations with loop dependent objects were mainly supported on geometric (rather than analytic) basis. For example, the geometric properties of the smoothened loops (i.e. the absence of kinks and intersections) underlies the topological construct (weaves) that bridges the usual metric tensor with the discrete properties of spacetime at the Planck length 10]. The solution obtained by Brügmann et al. was the first analytic (in the sense that it does not depend of any particular geometric property of the loops) and also the first to correspond to a nondegenerate state. Up today no others solutions of this type have been found.

The loop calculus faces up to significant difficulties at the analytic level that, for quantum gravity, have two features. For one hand, the knowledge of the analytic properties of the "kinematical" space of states (the knots) is very limited. On the other, any explicit analytic evaluation of the Hamiltonian constraint over diffeomorphism invariant loop wavefunctions involves hard technical and conceptual problems. The technical difficulties refer to both formal and regulated calculations. The conceptual ones concern the interpretation of a derivative operator over topological objects (see [11] for a discussion of this last point). This means that, in spite of the loop representation has had the virtue to unlock the canonical quantization program of gravity, the identification of the space of states of the theory in terms of functional of loops is at present far to be complete.

Extended loops offer a new avenue to this problem. The extended loops were introduced as suitable generalizations of ordinary loops (the usual group of loops is embedded into a local infinite dimensional Lie group, the extended loop group [12]). In resemblance with ordinary loops, an extended loop representation of any Lie-algebra valued connection theory can be constructed. In particular, the extended loop representation of the Ashtekar connection was recently developed [13, 14. This representation contains (and it is simply related 
to) the conventional loop representation of quantum gravity. As the extended loop group has a more rich mathematical structure than the usual group of loops, some benefits at the calculation and regularization levels are exhibited by the new representation. These advantages has been proved to be relevant in the study of the Wheeler-DeWitt equation [15, 16, 17].

The aim of this article is to develop a systematic method to obtain analytic expressions of diffeomorphism invariants (the extended knots). The general ideas of the method were advanced in [18]. Now we are going to study with some detail the mechanism of operation of the diffeomorphism constraint in terms of extended loops, and we will establish an explicit procedure to get solutions. Extended knots reduce to ordinary knots by means of a simple prescription (at least at the formal level). The application and potential significance of the method to the case of quantum gravity are discussed.

The article is organized as follows: in Sect. 2 the properties of the extended loop wavefunctions are considered from a general point of view. In Sect. 3 the rudiments of a method to construct analytic expressions of diffeomorphism invariants in terms of extended loops are introduced. The existence of a systematic for the operation of the constraints in the extended loop framework was first advanced in 16]. In Sect. 4 this systematic is applied to build up families of extended knots invariants. We define a family as a set of diffeomorphism invariants with a specific structure. In Sect. 5 we consider a particular family. The analytic expressions of the members of this family are derived and their identification with known knot invariants is performed. The Mandelstam identities of the members of the family are also analyzed. The implications of the results for quantum gravity are discussed in Sect. 7. This Section also includes the conclusions. An appendix with some useful results is added.

\section{Extended loop wavefunctions}

Ordinary loops can be codified by an infinite set of distributional fields, the multitangent fields. The multitangent fields are defined by the integration of an ordered sequence of distributions along a loop $\gamma$ in the following way

$$
X^{\mu_{1} \ldots \mu_{r}}(\gamma):=\oint_{\gamma} d y_{r}^{a_{r}} \ldots \oint_{\gamma} d y_{1}^{a_{1}} \delta\left(x_{r}-y_{r}\right) \ldots \delta\left(x_{1}-y_{1}\right) \Theta_{\gamma}\left(o, y_{1}, \ldots, y_{r}\right)
$$

where the $\Theta$ function orders the points of integration along the loop. The Greek indices represent paired vector and space indices $\left(\mu_{i} \equiv a_{i} x_{i}\right)$. The holonomy or

phase factor associated with a Lie-algebra valued connection can be written in terms of the multitangents in the form

$$
H_{A}(\gamma)=\sum_{r=0}^{\infty} A_{\mu_{1}} \ldots A_{\mu_{r}} X^{\mu_{1} \ldots \mu_{r}}(\gamma) \equiv A_{\boldsymbol{\mu}} X^{\boldsymbol{\mu}}(\gamma)
$$


where $\boldsymbol{\mu}:=\mu_{1} \ldots \mu_{r}$ represents a set of indices of rank $r(\boldsymbol{\mu})=r$ and $A_{\boldsymbol{\mu}} \equiv$ $A_{a_{1}}\left(x_{1}\right) \cdots A_{a_{r}}\left(x_{r}\right)$. In (2) a generalized Einstein convention is assumed (repeated bold Greek indices indicate a sum of the sets of indices from rank zero to infinity|). According to (2), all the information about loops necessary for the construction of the loop representation is concentrated into the entire set of multitangent fields. As far as the loop representation is concerned, the infinite string of multivector fields can be considered then as totally equivalent to the loop itself:

$$
\gamma \leftrightarrow \mathbf{X}(\gamma):=\left\{X^{\boldsymbol{\mu}}(\gamma) ; r(\boldsymbol{\mu})=0, \cdots, \infty\right\}
$$

Extended loops are generalizations of the multitangent fields to include more general fields. They have the general form

$$
\mathbf{X}:=\delta_{\nu} X^{\nu}
$$

where $[\mathbf{X}]^{\boldsymbol{\mu}} \equiv \delta_{\nu}^{\boldsymbol{\mu}} X^{\boldsymbol{\nu}}=X^{\boldsymbol{\mu}}$ are now $r(\boldsymbol{\mu})$-times "contravariant" generalized multitensors. $\delta_{\nu}^{\mu}$ acts as a diagonal (infinite dimensional) identity matrix $]$ and the "covariant" multivectors $\delta_{\boldsymbol{\nu}}$ can be viewed as a canonical basis for the extended loop vectors.

Any (well behaved) application $f:\{\mathbf{X}\} \rightarrow \mathcal{C}(\mathcal{R})$ from the extended loop space to complex (or real) numbers would define a suitable functional of extended loops. In the extended loop representation the wavefunctions are linear functionals of the multivector density fields $X^{\mu_{1} \ldots \mu_{r}}$ :

$$
\Psi(\mathbf{X})=\Psi_{\mu_{1} \ldots \mu_{r}} X^{\mu_{1} \ldots \mu_{r}}
$$

The propagators $\Psi_{\mu_{1} \ldots \mu_{r}}$ would characterize completely the linear wavefunction and they could satisfy specific symmetry requirements. In the case of quantum gravity, the extended loop wavefunctions can be viewed as formally given by the transform of wavefunctions with support in the space of connections:

$$
\psi(\mathbf{X})=\int D A W_{X}[A] \psi[A]
$$

where $W_{X}[A]:=\operatorname{Tr}\left[H_{A}(\mathbf{X})\right]=\operatorname{Tr}\left[A_{\boldsymbol{\mu}}\right] X^{\boldsymbol{\mu}}$ is the extended Wilson functional (an overcomplete basis for the extended loop transform) 1 . This means that the propagators of the wavefunctions associated with the quantum states of gravity can be formally written in the following way

$$
\psi_{\mu_{1} \ldots \mu_{r}}:=\int D A \psi[A] \operatorname{Tr}\left[A_{\mu_{1} \ldots \mu_{r}}\right]
$$

\footnotetext{
${ }^{1}$ We are using the convention $A_{\mu_{k+1} \ldots \mu_{k}} \equiv \mathbf{1}$ (the identity matrix) and $X^{\mu_{k+1} \ldots \mu_{k}} \equiv 1$.

${ }^{2}$ The diagonal elements are given by the product of generalized delta functions: $\delta_{\nu_{1}}^{\mu_{1}} \cdots \delta_{\nu_{r}}^{\mu_{r}}$, being $\delta_{\nu_{i}}^{\mu_{i}}:=\delta_{b_{i}}^{a_{i}} \delta\left(x_{i}-y_{i}\right), \mu_{i}=a_{i} x_{i}$ and $\nu_{i}=b_{i} y_{i}$.

${ }^{3}$ Notice the close resemblance between the above formal definitions with those of the conventional loop representation.
} 
This expression shows that the quantities $\psi_{\mu_{1} \ldots \mu_{r}}$ would inherit a set of identities related to the traces of Lie-algebra matrices (Mandelstam's type identities). For the case of the $S U(2)$ Ashtekar connection we have the following glue-property for the product of traces:

$$
\operatorname{Tr}\left[A_{\boldsymbol{\mu}}\right] \operatorname{Tr}\left[A_{\boldsymbol{\nu}}\right]=\operatorname{Tr}\left[A_{\boldsymbol{\mu \nu}}\right]+\operatorname{Tr}\left[A_{\boldsymbol{\mu}}\right]
$$

with $\overline{\boldsymbol{\mu}}:=(-1)^{n} \boldsymbol{\mu}^{-1}$ and $\boldsymbol{\mu}^{-1}:=\mu_{r} \ldots \mu_{1}$. Using the cyclicity of the trace and $(\mathbb{8})$ it is straightforward to derive the following identities:

$$
\begin{aligned}
\psi_{\boldsymbol{\mu}} & =\psi_{(\boldsymbol{\mu})_{c}} \\
\psi_{\boldsymbol{\mu}} & =\psi_{\overline{\boldsymbol{\mu}}} \\
\psi_{\boldsymbol{\alpha} \boldsymbol{\beta} \boldsymbol{\pi}}+\psi_{\boldsymbol{\alpha} \boldsymbol{\beta} \overline{\boldsymbol{\pi}}} & =\psi_{\boldsymbol{\beta} \boldsymbol{\alpha} \boldsymbol{\pi}}+\psi_{\boldsymbol{\beta} \boldsymbol{\alpha} \overline{\boldsymbol{\pi}}}
\end{aligned}
$$

We are going to see that the above set of symmetry properties reduce to the usual Mandelstam identities for loop wavefunctions when extended loops are particularized to ordinary loops. The specification to ordinary loops is made by substituting the general multivector fields by the multitangent fields:

$$
\psi(\mathbf{X}) \rightarrow \psi(\gamma)=\psi_{\boldsymbol{\mu}} X^{\boldsymbol{\mu}}(\gamma)
$$

This prescription puts into correspondence any linear extended loop wavefunction with a loop wavefunction (the converse is not true in general). The transcription to the case of the conventional loop representation is then accomplished by using the following group properties:

$$
\begin{aligned}
{\left[X\left(\gamma_{1}\right) \times X\left(\gamma_{2}\right)\right]^{\boldsymbol{\mu}} } & :=\delta_{\boldsymbol{\pi} \boldsymbol{\theta}}^{\boldsymbol{\mu}} X\left(\gamma_{1}\right)^{\boldsymbol{\pi}} X\left(\gamma_{2}\right) \\
X^{\overline{\boldsymbol{\mu}}}(\gamma) & =X^{\boldsymbol{\mu}}(\bar{\gamma})
\end{aligned}
$$

where $\times$ is the extended group product, $\gamma_{1} \gamma_{2}$ is the group composition in the nonparametric loop space and $\bar{\gamma}$ is the rerouted loop. Then we can write

$$
\begin{aligned}
& \psi\left(\gamma_{1} \gamma_{2}\right)=\psi_{\boldsymbol{\mu}} \delta_{\boldsymbol{\alpha} \boldsymbol{\beta}}^{(\boldsymbol{\mu})_{c}} X^{\boldsymbol{\alpha}}\left(\gamma_{1}\right) X^{\boldsymbol{\beta}}\left(\gamma_{2}\right)=\psi\left(\gamma_{2} \gamma_{1}\right) \\
& \psi(\gamma)=\psi_{\overline{\boldsymbol{\mu}}} X^{\boldsymbol{\mu}}(\gamma)=\psi_{\boldsymbol{\mu}} X^{\overline{\boldsymbol{\mu}}}(\gamma)=\psi(\bar{\gamma}) \\
& \psi\left(\gamma_{1} \gamma_{2} \gamma_{3}\right)=\psi_{\boldsymbol{\alpha} \boldsymbol{\beta} \boldsymbol{\pi}} X^{\boldsymbol{\alpha}}\left(\gamma_{1}\right) X^{\boldsymbol{\beta}}\left(\gamma_{2}\right) X^{\boldsymbol{\pi}}\left(\gamma_{3}\right) \\
& =\left[\psi_{\boldsymbol{\beta} \boldsymbol{\alpha} \boldsymbol{\pi}}+\psi_{\boldsymbol{\beta} \boldsymbol{\alpha} \overline{\boldsymbol{\pi}}}-\psi_{\boldsymbol{\alpha} \boldsymbol{\beta} \overline{\boldsymbol{\pi}}}\right] X^{\boldsymbol{\alpha}}\left(\gamma_{1}\right) X^{\boldsymbol{\beta}}\left(\gamma_{2}\right) X^{\boldsymbol{\pi}}\left(\gamma_{3}\right) \\
& =\psi\left(\gamma_{2} \gamma_{1} \gamma_{3}\right)+\psi\left(\gamma_{2} \gamma_{1} \overline{\gamma_{3}}\right)-\psi\left(\gamma_{1} \gamma_{2} \overline{\gamma_{3}}\right)
\end{aligned}
$$

Equations (15)-(17) are the usual Mandelstam identities for loop wavefunctions. As we have shown they can be viewed as particular cases of the symmetry identities (9)-(11). 
We review now the transformation properties of the extended loop wave-

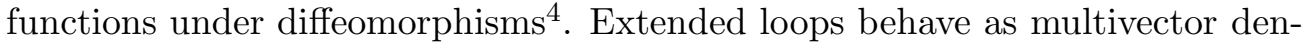
sities and the linear extended loop wavefunctions change under infinitesimal coordinate transformations $x^{\varsigma a}=x^{a}+\eta^{a}(x)$ in the way

$$
\psi\left(\mathbf{X}^{\prime}\right)=\psi(\mathbf{X})+\eta^{a x} \mathcal{C}_{a x} \psi(\mathbf{X})
$$

where

$$
\mathcal{C}_{a x}:=\left[\mathcal{F}_{a b}(x) \times \mathbf{X}^{(b x)}\right]^{\boldsymbol{\mu}} \frac{\delta}{\delta X^{\boldsymbol{\mu}}} \equiv \mathcal{F}_{a b}^{\boldsymbol{\alpha}}(x) X^{(b x \boldsymbol{\beta})_{c}} \frac{\delta}{\delta X^{\boldsymbol{\alpha} \boldsymbol{\beta}}}
$$

This result can be derived from the generator of diffeomorphisms in the connection space (via the extended loop transform) [14] or by using the explicit transformation properties of extended loops under infinitesimal coordinate transformations [20]. In (19), $\left[\mathbf{X}^{(b x)}\right]^{\boldsymbol{\beta}}:=X^{(b x \boldsymbol{\beta})_{c}}$ is the one-point-X (c indicates the average under cyclic permutation) and

$$
\mathcal{F}_{a b}^{\alpha_{1} \ldots \alpha_{r}}(x):=\epsilon_{a b c}\left[-\delta_{\nu_{1}}^{\alpha_{1} \ldots \alpha_{r}} g^{c x \nu_{1}}+\delta_{\nu_{1} \nu_{2}}^{\alpha_{1} \ldots \alpha_{r}} \epsilon^{c x \nu_{1} \nu_{2}}\right]
$$

$g^{c x \nu_{1}}$ is the inverse of the two point propagator of the Chern-Simons theory,

$$
g^{c x \nu_{1}}:=\epsilon^{c b_{1} k} \partial_{k} \delta\left(x-y_{1}\right)
$$

and

$$
\epsilon^{c x \nu_{1} \nu_{2}}:=\epsilon^{c b_{1} b_{2}} \delta\left(x-y_{1}\right) \delta\left(x-y_{2}\right)
$$

In the last expressions a mixed notation of Greek and paired indices were used. Let us consider now the specification of (18) to the case of ordinary loops. From (5) and (19) we can write

$$
\begin{aligned}
\mathcal{C}_{a x} \psi[\mathbf{X}(\gamma)] & =\psi_{\boldsymbol{\alpha} \boldsymbol{\pi} \boldsymbol{\theta}} \mathcal{F}_{a b}^{\boldsymbol{\alpha}}(x) X^{\boldsymbol{\theta} b x} \boldsymbol{\pi}_{(\gamma)} \\
& =\oint_{\gamma} d y^{b} \delta(x-y) \psi_{\boldsymbol{\alpha} \boldsymbol{\pi} \boldsymbol{\theta}} \mathcal{F}_{a b}^{\boldsymbol{\alpha}}(y) X^{\boldsymbol{\pi}}\left(\gamma_{y}^{o}\right) X^{\boldsymbol{\theta}}\left(\gamma_{o}^{y}\right) \\
& =\oint_{\gamma} d y^{b} \delta(x-y) \psi_{\boldsymbol{\mu}}\left[\mathcal{F}_{a b}(x) \times \mathbf{X}\left(\gamma_{y}^{o}\right) \times \mathbf{X}\left(\gamma_{o}^{y}\right)\right]^{\boldsymbol{\mu}}
\end{aligned}
$$

where $\gamma_{o}^{y}$ is an open path form $o$ to $y$. Introducing now the group identity $1=\mathbf{X}\left(\bar{\gamma}_{o}^{y}\right) \times \mathbf{X}\left(\gamma_{o}^{y}\right)$ and using the cyclicity of the set of indices $\boldsymbol{\mu}$ we get

$$
\begin{aligned}
\mathcal{C}_{a x} \psi(\gamma) & =\oint_{\gamma} d y^{b} \delta(x-y) \psi_{\boldsymbol{\mu}}\left[\mathbf{X}\left(\gamma_{o}^{y}\right) \times \mathcal{F}_{a b}(y) \times \mathbf{X}\left(\bar{\gamma}_{o}^{y}\right) \times \mathbf{X}\left(\gamma_{o}^{y}\right) \times \mathbf{X}\left(\gamma_{y}^{o}\right)\right]^{\boldsymbol{\mu}} \\
& \equiv \oint_{\gamma} d y^{b} \delta(x-y) \Delta_{a b}\left(\gamma^{y}\right) \psi(\gamma)
\end{aligned}
$$

\footnotetext{
${ }^{4}$ The properties of extended objects under general coordinate transformations were first considered by [19] for multitangent fields and later by 12 for extended loops.
} 
where

$$
\Delta_{a b}\left(\gamma^{y}\right) \mathbf{X}(\gamma):=\left[\mathbf{X}\left(\gamma_{o}^{y}\right) \times \mathcal{F}_{a b}(y) \times \mathbf{X}\left(\bar{\gamma}_{o}^{y}\right)\right] \times \mathbf{X}(\gamma)
$$

is the loop derivative in the non-parametric loop space [21]. The expression (24) gives the correct transformation law of loop wavefunctions under diffeomorphisms.

\section{$3 \quad$ Extended knot invariants}

The previous discussion shows that the solutions of the equation

$$
\mathcal{C}_{a x} \psi(\mathbf{X})=0
$$

can be viewed as defining "extended knot invariants" (in the sense that the restriction of the domain of definition of the solution to ordinary loops will always gives a knot invariant). The analysis of this equation in the extended loop space reveals the existence of a specific mechanism linking the action of the operator with some definite structure of the propagators $\psi_{\boldsymbol{\mu}}$. To see this we write (26) in the form

$$
\mathcal{C}_{a x} \psi(\mathbf{X})=\sum_{r=0}^{\infty} \epsilon_{a b c} \psi_{\mu_{1} \ldots \mu_{r}}\left[-g^{c x \mu_{1}} X^{\left(b x \mu_{2} \ldots \mu_{r}\right)_{c}}+\epsilon^{c x \mu_{1} \mu_{2}} X^{\left(b x \mu_{3} \ldots \mu_{r}\right)_{c}}\right]=0
$$

The possibility to systematize the search of solutions of (27) is based on the following two punctuations:

P1: The propagators $\psi_{\mu_{1} \ldots \mu_{r}}$ are expressed completely in terms of the two $(g .$. and three $(h \ldots)$ point propagators of the Chern-Simons theory.

P2: The propagators $\psi_{\mu_{1} \ldots \mu_{r}}$ exhibit a definite skeleton structure in terms of the basic "blocks" $g$ and $h$.

The architectonic profile of the skeleton is:

S1: The propagators vanish for a certain (maximum) rank. That is to say, $\psi_{\mu_{1} \ldots \mu_{r}}=0$ for all $r>N$.

S2: For $r=N, \psi_{\mu_{1} \ldots \mu_{N}}$ is expressed entirely in terms of the two point ChernSimons propagator. Specifically, they are cyclic combinations of products of $g$ 's.

S3: For the successive decreasing ranks the "free" $g$ 's are progressively substituted by "connected" $h$ 's. The final expression is always cyclic in the covariant Greek indices.

S4: The minimum rank $n$ does not contain any free two point Chern-Simons propagator. 
The above nomenclature of free- $g$ and connected- $h$ refers to the analytic properties of the Chern-Simons propagators. In fact, the three point propagator $h_{\mu_{1} \mu_{2} \mu_{3}}$ is nothing else than the "contraction" of three free two point $g_{\mu_{i} \alpha_{i}}$ with the "vertex" $\epsilon^{\alpha_{1} \alpha_{2} \alpha_{3}}$ :

$$
h_{\mu_{1} \mu_{2} \mu_{3}}:=\epsilon^{\alpha_{1} \alpha_{2} \alpha_{3}} g_{\mu_{1} \alpha_{1}} g_{\mu_{2} \alpha_{2}} g_{\mu_{3} \alpha_{3}}
$$

The free propagator can be written in the following way,

$$
g_{\mu_{1} \mu_{2}} \equiv \epsilon_{a_{1} a_{2} c} \phi_{x_{2}}^{c x_{1}}:=-\epsilon_{a_{1} a_{2} c} \frac{\partial^{c}}{\nabla^{2}} \delta\left(x_{1}-x_{2}\right)
$$

The function $\phi$ plays a prominent role in the extended loop formalism. It fixes a transverse prescription for the multivector fields through the following decomposition of the identity: $\delta_{\nu_{1}}^{\mu_{1}}=\delta_{\mathrm{T}}{ }^{\mu_{1}}{ }_{\nu_{1}}+\phi_{z_{1}, d_{1}}^{\mu_{1}}$. Then

$$
Y^{\mu_{1}}=\delta_{\mathrm{T}}^{\mu_{1}}{ }_{\nu_{1}} X^{\nu_{1}}
$$

is a transverse (divergence free) field. This property can be directly generalized to the case of multivector fields [12]. Notice that

$$
\mathcal{F}_{a b}^{\mu_{1}} g_{\mu_{1} \mu_{2}}=-\epsilon_{a b c} \delta_{\mathrm{T}}^{c x}{ }_{\mu_{2}}
$$

whereas

$$
\mathcal{F}_{a b}^{\mu_{1}} h_{\mu_{1} \mu_{2} \mu_{3}}=-\epsilon_{a b c} \delta_{\mathrm{T}}{ }^{c x}{ }_{\alpha_{1}} \epsilon^{\alpha_{1} \alpha_{2} \alpha_{3}} g_{\mu_{2} \alpha_{2}} g_{\mu_{3} \alpha_{3}}
$$

with

$$
\delta_{\mathrm{T}}^{c x}{ }_{\alpha_{1}} \epsilon^{\alpha_{1} \alpha_{2} \alpha_{3}}=\epsilon^{c x \alpha_{2} \alpha_{3}}+\left(\phi_{z_{2}}^{c x}-\phi_{z_{3}}^{c x}\right) g^{\alpha_{2} \alpha_{3}}
$$

being $z_{k}$ the spatial part of the indices $\alpha_{k}$. Let us return to the skeleton rules S1-S4. They mirror the analytic properties of knot invariants known at present?. A point to stress is that no other propagators than the Chern-Simons are known to participate in the analytic properties of knots. In our case the rules S1-S4 provide the first ingredient for a systematization: the propagators $\psi_{\mu_{1} \ldots \mu_{r}}$ are structured according a sequential arrangement of some basic blocks in the form

$$
\psi(\mathbf{X})=g_{. .} g_{. .} \cdots g_{. .} X^{\cdots \cdots \cdots}+h_{\ldots} \cdots g_{. .} X^{\cdots \cdots \cdots}+\cdots+h_{\ldots} \cdots h_{\ldots} X^{\cdots \cdots}
$$

The second ingredient appears when (27) (and in particular (31) and (32)) start to work. The following general observations can be outlined from the action of the diffeomorphism operator over wavefunctions of the type (34):

\footnotetext{
${ }^{5}$ The state given by the exponential of the self-linking number is an exception to S1. The breakdown of S1 (i.e. the appearance of series) introduces convergence problems into the formalism. The consideration of this question is out of the scope of the present discussion.
} 
O1: The successive ranks of the wavefunction are linked intimately. As a general rule, $\mathcal{C}_{a x}$ acting on a generic rank $r$ produces two types of contributions, one of rank $r$ and other of rank $r-1$. For $r>n$, the contribution of rank $r-1$ is always canceled by terms that appear when the operator act on the rank $r-1$ of the wavefunction. A chain of cancellations is then induced by the action of the diffeomorphism operator?.

O2: For $n<r \leq N$ there exist in general remnant terms that do not enter into the chain of cancellations. The general form of these terms is $\epsilon_{a b c} \psi \ldots . . X^{(b x \ldots c x \ldots)_{c}}$ and they have to vanish by means of symmetry considerations 7 .

O3: The term of lower rank $n$ is responsible of the closure of the chain in a consistent way.

In what follows we are going to use the above punctuations as guidelines to establish a systematic method to construct extended knot invariants.

\section{Extended knot families}

We start by describing in general the properties of the final product we get: the extended knot families. They are given by sets $\left\{\psi_{i}\right\}_{n}^{N}$ of linear extended loop wavefunctions with the same maximum and minimum ranks that satisfy the following conditions:

F1: $\mathcal{C}_{a x} \psi_{i}=0$ for all $i$.

F2: $\psi_{i}(\mathbf{X})=\psi^{i} \boldsymbol{\mu} X^{\boldsymbol{\mu}}$, with $\psi^{i} \boldsymbol{\mu}$ a cyclic propagator that fulfill the skeleton rules S1-S4.

F3: $\psi^{i} \mu_{1} \ldots \mu_{n}$ is the same for all members of the family.

\subsection{The maximum (even) rank}

For $r=N$ the propagators $\psi^{i}{ }_{\mu_{1} \ldots \mu_{N}}$ are written as cyclic combinations of products of $g$ 's. For $N=6$ we have for example the following possibilities:

$$
\begin{aligned}
C^{1}{ }_{\mu_{1} \ldots \mu_{6}}= & g_{\mu_{1} \mu_{4}} g_{\mu_{2} \mu_{5}} g_{\mu_{3} \mu_{6}} \\
C^{2}{ }_{\mu_{1} \ldots \mu_{6}}= & g_{\mu_{1} \mu_{2}} g_{\mu_{3} \mu_{4}} g_{\mu_{5} \mu_{6}}+g_{\mu_{1} \mu_{6}} g_{\mu_{2} \mu_{3}} g_{\mu_{4} \mu_{5}} \\
C^{3}{ }_{\mu_{1} \ldots \mu_{6}}= & g_{\mu_{1} \mu_{2}} g_{\mu_{3} \mu_{6}} g_{\mu_{4} \mu_{5}}+g_{\mu_{1} \mu_{4}} g_{\mu_{2} \mu_{3}} g_{\mu_{5} \mu_{6}}+g_{\mu_{1} \mu_{6}} g_{\mu_{2} \mu_{5}} g_{\mu_{3} \mu_{4}} \\
C^{4}{ }_{\mu_{1} \ldots \mu_{6}}= & g_{\mu_{1} \mu_{3}} g_{\mu_{2} \mu_{5}} g_{\mu_{4} \mu_{6}}+g_{\mu_{1} \mu_{4}} g_{\mu_{2} \mu_{6}} g_{\mu_{3} \mu_{5}}+g_{\mu_{1} \mu_{5}} g_{\mu_{2} \mu_{4}} g_{\mu_{3} \mu_{6}} \\
C^{5}{ }_{\mu_{1} \ldots \mu_{6}}= & g_{\mu_{1} \mu_{2}} g_{\mu_{3} \mu_{5}} g_{\mu_{4} \mu_{6}}+g_{\mu_{1} \mu_{3}}\left(g_{\mu_{2} \mu_{6}} g_{\mu_{4} \mu_{5}}+g_{\mu_{2} \mu_{4}} g_{\mu_{5} \mu_{6}}\right)+ \\
& g_{\mu_{1} \mu_{6}} g_{\mu_{2} \mu_{4}} g_{\mu_{3} \mu_{5}}+g_{\mu_{1} \mu_{5}}\left(g_{\mu_{2} \mu_{3}} g_{\mu_{4} \mu_{6}}+g_{\mu_{2} \mu_{6}} g_{\mu_{3} \mu_{4}}\right)
\end{aligned}
$$

\footnotetext{
${ }^{6}$ Exactly the same chain of cancellations takes place in the case of the Hamiltonian. This is a characteristic of the extended loop representation, see [16].

${ }^{7}$ For extended knots this contribution involves in general a symmetric expression in the indices $b x$ and $c x$.
} 
We need some suitable notation in order to reflect appropriately the action of the operator $\mathcal{C}_{a x}$. Putting

$$
\psi^{i}{ }_{\mu_{1} \ldots \mu_{N}}=\sum_{[k]} g_{\mu_{1} \mu_{k}} G^{i} \mu_{2} \ldots \mu_{k-1} \mu_{k+1} \ldots \mu_{N}
$$

we found

$$
\begin{array}{r}
\left.\mathcal{C}_{a x} \psi_{i}\right|_{r=N}=-\sum_{[k]} \epsilon_{a b c} \delta_{\mathrm{T}}^{c x}{ }_{\mu_{k}} G^{i}{ }_{\mu_{2} \ldots \mu_{k-1} \mu_{k+1} \ldots \mu_{N}} X^{\left(b x \mu_{2} \ldots \mu_{N}\right)_{c}} \\
+\sum_{[k, l]} g_{\mu_{k}[a x} g_{b x] \mu_{l}} G^{i}{ }_{\mu_{3} \ldots \mu_{k-1} \mu_{k+1} \ldots \mu_{l-1} \mu_{l+1} \ldots \mu_{N}} X^{\left(b x \mu_{3} \ldots \mu_{N}\right)_{c}}
\end{array}
$$

Using the fact that $\delta_{\mathrm{T}}=\delta-\phi$ and integrating by parts the longitudinal projector contribution we obtain

$$
\begin{aligned}
& \left.\mathcal{C}_{a x} \psi_{i}\right|_{r=N}=-\sum_{[k]} \epsilon_{a b c} G^{i}{ }_{\mu_{2} \ldots \mu_{k-1} \mu_{k+1} \ldots \mu_{N}} X^{\left(b x \mu_{2} \ldots \mu_{k-1} c x \mu_{k+1} \ldots \mu_{N}\right)_{c}} \\
& -\sum_{[k]} \epsilon_{a b c}\left(\phi_{x_{k-1}}^{c x}-\phi_{x_{k+1}}^{c x}\right) G_{\mu_{2} \ldots \mu_{k-1} \mu_{k+1} \ldots \mu_{N}}^{i} X^{\left(b x \mu_{2} \ldots \mu_{k-1} \mu_{k+1} \ldots \mu_{N}\right)_{c}} \\
& +\sum_{[k, l]} g_{\mu_{k}[a x} g_{b x] \mu_{l}} G^{i} \mu_{3 \ldots \mu_{k-1} \mu_{k+1} \ldots \mu_{l-1} \mu_{l+1} \ldots \mu_{N}} X^{\left(b x \mu_{3} \ldots \mu_{N}\right)_{c}}
\end{aligned}
$$

where we have used the differential constraint [12] of the multivector fields. We get three contributions, the first of rank $N$ and the others of rank $N-1$. It is easy to see that a sufficient condition for the contribution of rank $N$ to vanish is the cyclicity of $\psi_{\mu_{1} \ldots \mu_{N}}{ }^{\text {? }}$.

\subsection{The rank $N-1$}

The next rank is formed by substituting two $g$ 's for one $h$ 甲. For $N-1=5$ one finds two cyclic combinations of this type

$$
\begin{aligned}
C^{1}{ }_{\mu_{1} \ldots \mu_{5}} & =g_{\left(\mu_{1} \mu_{2}\right.} h_{\left.\mu_{3} \mu_{4} \mu_{5}\right)_{c}} \\
C^{2}{ }_{\mu_{1} \ldots \mu_{5}} & =g_{\left(\mu_{1} \mu_{3}\right.} h_{\left.\mu_{2} \mu_{4} \mu_{5}\right)_{c}}
\end{aligned}
$$

Now we have to distinguish when $\mu_{1}$ belongs to a $g$ or to an $h$. Writing

\footnotetext{
${ }^{8}$ I thank C. Di Bartolo to point me out this fact.

${ }^{9}$ There is not a unique way to make this passage guided only by S3. For example for $N=10$ we have two possibilities: $h_{\mu_{1} \mu_{2} \mu_{3}} g_{\mu_{4} \mu_{5}} g_{\mu_{6} \mu_{7}} g_{\mu_{8} \mu_{9}}$ or $h_{\mu_{1} \mu_{2} \mu_{3}} h_{\mu_{4} \mu_{5} \mu_{6}} h_{\mu_{7} \mu_{8} \mu_{9}}$. In order to simplify the discussion we shall limit to consider only the first case.
} 


$$
\begin{aligned}
& \psi_{\mu_{1} \ldots \mu_{N-1}}^{i}=\sum_{[k]} g_{\mu_{1} \mu_{k}} H^{i}{ }_{\mu_{2} \ldots \mu_{k-1} \mu_{k+1} \ldots \mu_{N-1}} \\
& \quad+\sum_{[k, l]} h_{\mu_{1} \mu_{k} \mu_{l}} G^{i}{ }_{\mu_{2} \ldots \mu_{k-1} \mu_{k+1} \ldots \mu_{l-1} \mu_{l+1} \ldots \mu_{N-1}}
\end{aligned}
$$

we get

$$
\begin{aligned}
\mathcal{C}_{a x} & \psi_{i} \\
\left.\right|_{r=N-1} & =-\sum_{[k]} \epsilon_{a b c} H_{\mu_{2} \ldots \mu_{k-1} \mu_{k+1} \ldots \mu_{N-1}}^{i} X^{\left(b x \mu_{2} \ldots \mu_{k-1} c x \mu_{k+1} \ldots \mu_{N-1}\right)_{c}} \\
- & \sum_{[k, l]} \epsilon_{a b c}\left(\phi_{x_{l}}^{c x}-\phi_{x_{k}}^{c x}\right) g_{\mu_{k} \mu_{l}} G_{\mu_{3} \ldots \mu_{k-1} \mu_{k+1} \ldots \mu_{l-1} \mu_{l+1} \ldots \mu_{N}}^{i} X^{\left(b x \mu_{3} \ldots \mu_{N}\right)_{c}} \\
- & \sum_{[k, l]} g_{\mu_{k}[a x} g_{b x] \mu_{l}} G^{i}{ }_{\mu_{3} \ldots \mu_{k-1} \mu_{k+1} \ldots \mu_{l-1} \mu_{l+1} \ldots \mu_{N}} X^{\left(b x \mu_{3} \ldots \mu_{N}\right)_{c}} \\
& + \text { terms of rank } N-2
\end{aligned}
$$

The comparison of (42) and (46) permits to conclude that: 1) the terms of the type described in $\mathrm{O} 2$ appear whenever the $\mu_{1}$ index lies in a free- $g$. 2) The presence of the $h$ in the rank $N-1$ of the wavefunction is responsible for the generation of terms with exactly the form needed to cancel all the contribution of rank $N-1$ in (42). In other worlds, the connected- $h$ starts the chain of cancellations associated with the diffeomorphism operator.

As in the case of rank $N$, the cyclicity of $\psi_{\mu_{1} \ldots \mu_{N-1}}$ assures the annulment of the first sum in (46). Then, if the wavefunction's propagators of ranks $N$ and $N-1$ are suitables to produce the cancellation of the remaining sums in (42) and (46) between themselves we can write

$$
\mathcal{C}_{a x}\left\{\left.\psi_{i}\right|_{r=N}+\left.\psi_{i}\right|_{r=N-1}\right\}=\text { terms of rank } N-2
$$

Due to the higher content of connected propagators as well as the multiplication of possibilities to go from one rank to the following, the analysis becomes more intricate as one advances along the chain. The main noticed characteristics are however preserved, so one can write in general

$$
\mathcal{C}_{a x}\left\{\left.\psi_{i}\right|_{r=N}+\left.\psi_{i}\right|_{r=N-1}+\cdots+\left.\psi_{i}\right|_{r=m}\right\}=\text { terms of rank } m-1
$$

\subsection{The minimum rank}

What happens in (48) when one gets the minimum rank? The only possibility is that the contribution of rank $n-1$ vanishes identically. This condition has a definite meaning that we clarify with a simple example. The minimum rank is composed only by connected propagators. The most simple case is a single $h$. One finds for this case 


$$
\begin{aligned}
\mathcal{C}_{a x} h_{\mu_{1} \mu_{2} \mu_{3}} X^{\mu_{1} \mu_{2} \mu_{3}}= & \left\{-g_{\mu_{1}[a x} g_{b x] \mu_{2}}+\epsilon_{a b c}\left(\phi_{x_{1}}^{c x}-\phi_{x_{2}}^{c x}\right) g_{\mu_{1} \mu_{2}}\right\} X^{\left(b x \mu_{1} \mu_{2}\right)_{c}} \\
& +2\left\{h_{a x b x \mu_{1}}-\epsilon_{a b c} \phi_{z}^{c x} \phi_{x}^{d z} g_{\mu_{1} d z}\right\} X^{\left(b x \mu_{1}\right)_{c}}
\end{aligned}
$$

where an integration in the spatial variable $z$ is assumed. Notice that we can not use here symmetry arguments of the type O2. The only general way to annul the contribution of rank 2 in (49) is by demanding

$$
\left\{h_{a x b x \mu_{1}}-\epsilon_{a b c} \phi_{z}^{c x} \phi_{x}^{d z} g_{\mu_{1} d z}\right\} \equiv 0
$$

But this is a formal identity for the propagators! Indeed, from (28) it is straightforward to see that the second term of the l.h.s. is a formal equivalent way to write the three point propagator with two spatial indices fixed at $x$. The annulment of the contribution of rank $n-1$ for $m=n$ in (48) appears then as a consistency condition for the closure of the chain.

\subsection{The method}

We have seen that the existence of a systematic procedure to obtain diffeomorphism invariants is suggested by the characteristics of the extended loop calculus. Also that the complexity of the analysis grows significantly at higher ranks. A practical way to proceed is the following:

a) Select a cyclic expression of Chern-Simons propagators that not contain any free $g$. Verify the consistence condition $O 3$ according the analysis of the preceding subsection. We get then a candidate for the minimum rank $n$.

b) Find the expressions of rank $n+1$ that make $\mathcal{C}_{a x}\left\{\left.\psi_{i}\right|_{r=n+1}+\left.\psi_{i}\right|_{r=n}\right\}=$ terms of rank $n+1$. Verify that the terms of the form $\epsilon_{a b c} \psi \ldots \ldots X^{(b x \ldots c x \ldots)_{c}}$ vanish by symmetry considerations.

c) Repeat the procedure until all the connected parts are substituted by free- $g$ 's.

By this procedure the extended knot families are generated.

\section{The family $\left\{\psi_{i}\right\}_{4}^{6}$}

We apply now the method to construct a family with maximum rank 6 and minimum rank 4 . For $n=4$

$$
C_{\mu_{1} \ldots \mu_{4}}=h_{\mu_{1} \mu_{2} \alpha} g^{\alpha \beta} h_{\beta \mu_{3} \mu_{4}}-h_{\mu_{1} \mu_{4} \alpha} g^{\alpha \beta} h_{\beta \mu_{2} \mu_{3}}
$$

is a suitable expression that not contains free- $g$ 's $\mathrm{s}^{\mathbb{0}}$. We get for this case (see [16]):

\footnotetext{
${ }^{10}$ Another more connected possibility is: $h_{\mu_{1} \alpha_{1} \alpha_{2}} g^{\alpha_{1} \beta_{1}} g^{\alpha_{2} \beta_{2}} h_{\beta_{1} \beta_{2} \mu_{2}} h_{\mu_{3} \alpha_{3} \alpha_{4}} g^{\alpha_{3} \beta_{3}} g^{\alpha_{4} \beta_{4}} h_{\beta_{3} \beta_{4} \mu_{4}}+$ cyclic permutations.
} 


$$
\begin{aligned}
& \mathcal{C}_{a x} C_{\mu_{1} \ldots \mu_{4}} X^{\mu_{1} \ldots \mu_{4}}=2\left\{g_{\mu_{1}[a x} h_{b x] \mu_{2} \mu_{3}}-\epsilon_{a b c} \phi_{x_{1}}^{c x} h_{\mu_{1} \mu_{2} \mu_{3}}\right. \\
& \left.\quad+\epsilon_{a b c} \phi_{z}^{c x}\left(\phi_{x_{3}}^{d z}-\phi_{x_{2}}^{d z}\right) g_{\mu_{1} d z} g_{\mu_{2} \mu_{3}}\right\} X^{\left(b x \mu_{1} \mu_{2} \mu_{3}\right)_{c}}+\mathcal{I}_{a x b x \mu_{1} \mu_{2}} X^{\left(b x \mu_{1} \mu_{2}\right)_{c}}
\end{aligned}
$$

with

$$
\begin{aligned}
& \mathcal{I}_{a x b x \mu_{1} \mu_{2}}:=-2 h_{a x b x \alpha} g^{\alpha \beta} h_{\mu_{1} \mu_{2} \beta}+h_{\mu_{2} \alpha[a x} h_{b x] \mu_{1} \beta} g^{\alpha \beta} \\
& \quad-2 \epsilon_{a b c} \phi_{z}^{c x}\left(\phi_{x}^{d z}-\phi_{x_{1}}^{d z}\right) h_{d z \mu_{1} \mu_{2}}-2 \epsilon_{a b c} \phi_{z}^{c x} \phi_{y}^{d z}\left(\phi_{x}^{e y}+\phi_{x_{1}}^{e y}\right) g_{\mu_{1} d z} g_{\mu_{2} \text { ey }}
\end{aligned}
$$

In reference [16] it was demonstrated that formally $\mathcal{I}_{a x b x \mu_{1} \mu_{2}} \equiv 0$, so the consistency condition is fulfilled. For the rank $n+1=5$ the propagators have the general form $\left.g_{(. .} h_{. .}\right)_{c}$. According the results listed in the Appendix it is easy to see that the combinations $C_{\mu_{1} \ldots \mu_{5}}^{1} X^{\mu_{1} \ldots \mu_{5}}-C_{\mu_{1} \ldots \mu_{4}} X^{\mu_{1} \ldots \mu_{4}}$ and $C_{\mu_{1} \ldots \mu_{5}}^{2} X^{\mu_{1} \ldots \mu_{5}}+C_{\mu_{1} \ldots \mu_{4}} X^{\mu_{1} \ldots \mu_{4}}$ satisfy the requirement $\left.b\right)$. The next rank would involve expressions of the form $g_{(. .} g_{. .} g_{. .)_{c}}$ and the procedure is completed. By using again the results of the Appendix one finds:

$$
\begin{aligned}
\psi_{1}= & {\left[C_{\mu_{1} \ldots \mu_{6}}^{2}+C_{\mu_{1} \ldots \mu_{6}}^{3}\right] X^{\mu_{1} \ldots \mu_{6}}-C_{\mu_{1} \ldots \mu_{5}}^{1} X^{\mu_{1} \ldots \mu_{5}}+C_{\mu_{1} \ldots \mu_{4}} X^{\mu_{1} \ldots \mu_{4}} } \\
\psi_{2}= & {\left[C_{\mu_{1} \ldots \mu_{6}}^{1}+C_{\mu_{1} \ldots \mu_{6}}^{4}+C_{\mu_{1} \ldots \mu_{6}}^{5}\right] X^{\mu_{1} \ldots \mu_{6}}+C_{\mu_{1} \ldots \mu_{5}}^{1} X^{\mu_{1} \ldots \mu_{5}} } \\
& -C_{\mu_{1} \ldots \mu_{4}} X^{\mu_{1} \ldots \mu_{4}} \\
\psi_{3}= & {\left[2 C_{\mu_{1} \ldots \mu_{6}}^{1}+C_{\mu_{1} \ldots \mu_{6}}^{4}\right] X^{\mu_{1} \ldots \mu_{6}}+C_{\mu_{1} \ldots \mu_{5}}^{2} X^{\mu_{1} \ldots \mu_{5}}+C_{\mu_{1} \ldots \mu_{4}} X^{\mu_{1} \ldots \mu_{4}} } \\
\psi_{4}= & {\left[-C_{\mu_{1} \ldots \mu_{6}}^{1}+C_{\mu_{1} \ldots \mu_{6}}^{2}+C_{\mu_{1} \ldots \mu_{6}}^{3}+C_{\mu_{1} \ldots \mu_{6}}^{5}\right] X^{\mu_{1} \ldots \mu_{6}}-C_{\mu_{1} \ldots \mu_{5}}^{2} X^{\mu_{1} \ldots \mu_{5}} } \\
& -C_{\mu_{1} \ldots \mu_{4}} X^{\mu_{1} \ldots \mu_{4}}
\end{aligned}
$$

No other analytic expressions of the general form $\psi=$ g.. $. . g_{. .} X^{\cdots \cdots}+g . . h \ldots X^{\cdots \cdots}+$ $h_{. \star \star} g^{\star \star} h_{\star . .} X^{\cdots \cdots}$ invariant under diffeomorphisms apart form that listed above would exist. Two immediate questions arise: 1) to which knot invariants would correspond the different members of the family $\left\{\psi_{i}\right\}_{4}^{6}$, and 2) which among them would satisfy the Mandelstam identities?

To answer the first question we need to introduce another player into scene: the $*$-product of diffeomorphism invariants [15, 17]. The $*$-product allows to put into correspondence linear invariant expressions with the usual product of diffeomorphism invariants. For example, the $*$-square of the Gauss invariant $\varphi_{G}:=g_{\mu_{1} \mu_{2}} X^{\mu_{1} \mu_{2}}$ is defined in the following way! $\square$

$$
\begin{aligned}
\left(* \varphi_{G}\right)^{2}:= & g_{\mu_{1} \mu_{2}} g_{\mu_{3} \mu_{4}} X^{\mu_{1} \mu_{2}} \mu_{3} \mu_{4} \\
= & g_{\mu_{1} \mu_{2}} g_{\mu_{3} \mu_{4}}\left(X^{\mu_{1} \mu_{2} \mu_{3} \mu_{4}}+X^{\mu_{1} \mu_{3} \mu_{2} \mu_{4}}+X^{\mu_{1} \mu_{3} \mu_{4} \mu_{2}}\right. \\
& \left.+X^{\mu_{3} \mu_{1} \mu_{2} \mu_{4}}+X^{\mu_{3} \mu_{1} \mu_{4} \mu_{2}}+X^{\mu_{3} \mu_{4} \mu_{1} \mu_{2}}\right) \\
\equiv & 2\left(g_{\mu_{1} \mu_{2}} g_{\mu_{3} \mu_{4}}+g_{\mu_{1} \mu_{3}} g_{\mu_{2} \mu_{4}}+g_{\mu_{1} \mu_{4}} g_{\mu_{2} \mu_{3}}\right) X^{\mu_{1} \mu_{2} \mu_{3} \mu_{4}} \\
&
\end{aligned}
$$

\footnotetext{
${ }^{11}$ The underline of an ordered subset $\mu_{1} \ldots \mu_{k} \mu_{k+1} \ldots \mu_{r}$ of $k$ indices indicates in general the linear combination of multivectors obtained by permuting the indices in all possible ways but preserving the relative order of the subsets $\mu_{1} \ldots \mu_{k}$ and $\mu_{k+1} \ldots \mu_{r}$ among themselves.
} 
For those extended loops that satisfy the algebraic constraint $X \underline{\mu_{1} \mu_{2}} \mu_{3} \mu_{4}=$ $X^{\mu_{1} \mu_{2}} X^{\mu_{3} \mu_{4}}$, (58) reduces to the usual product of Gauss invariants (this is a general property of the $*$-composition law). In general, the $*$-product of two diffeomorphism invariants is a diffeomorphism invariant [20]. From equations (54)-(57) the following relationships (constraints) between the members of the family are found:

$$
\begin{aligned}
\psi_{1}+\psi_{2}=\psi_{3}+\psi_{4} & =\frac{1}{3 !}\left(* \varphi_{G}\right)^{3} \\
\psi_{2}+\psi_{3} & =\varphi_{G} * J_{2}
\end{aligned}
$$

where $J_{2}:=g_{\mu_{1} \mu_{3}} g_{\mu_{2} \mu_{4}} X^{\mu_{1} \mu_{2} \mu_{3} \mu_{4}}+h_{\mu_{1} \mu_{2} \mu_{3}} X^{\mu_{1} \mu_{2} \mu_{3}}$ is the analytic part of the second coefficient of the Alexander-Conway knot polynomial (that coincides with the second coefficient of certain expansion of the Jones polynomial). We conclude that of the four members of the family, only one would represent a new diffeomorphism invariant. Taking this single invariant as $\psi_{3} \equiv J_{3}$, we get from $(59)-(60)$

$$
\begin{aligned}
& \psi_{1}=\frac{1}{3 !}\left(* \varphi_{G}\right)^{3}-\varphi_{G} * J_{2}+J_{3} \\
& \psi_{2}=\varphi_{G} * J_{2}-J_{3} \\
& \psi_{3}=J_{3} \\
& \psi_{4}=\frac{1}{3 !}\left(* \varphi_{G}\right)^{3}-J_{3}
\end{aligned}
$$

$\varphi_{G}$ and $J_{2}$ belong to lower rank families [18]:

$$
\begin{aligned}
& \left\{\psi_{i}\right\}_{2}^{2}=\left\{\varphi_{G}\right\} \\
& \left\{\psi_{i}\right\}_{3}^{4}=\left\{J_{2}, \frac{1}{2}\left(* \varphi_{G}\right)^{2}-J_{2}\right\}
\end{aligned}
$$

Using the above results the family $\left\{\psi_{i}\right\}_{4}^{6}$ can be written in the form

$$
\left\{\psi_{i}\right\}_{4}^{6}=\left\{K_{3}, \frac{1}{3 !}\left(* \varphi_{G}\right)^{3}-K_{3}, J_{3}, \frac{1}{3 !}\left(* \varphi_{G}\right)^{3}-J_{3}\right\}
$$

with

$$
K_{3}:=J_{3}-J_{2} * \varphi_{G}+\frac{1}{3 !}\left(* \varphi_{G}\right)^{3}
$$

$K_{3}$ is related to the third coefficient of certain expansion of the Kauffman bracket knot polynomial in terms of the cosmological constant [2], and $J_{3}$ corresponds to the analytic part of the third coefficient of a similar expansion of the Jones polynomial [22]. The identification of the members of the family is then accomplished. Notice that the family is composed in essence by those invariants involved (at third order) in the relationship

\footnotetext{
${ }^{12}$ If $K_{\Lambda}:=\tilde{K}_{n} \Lambda^{n}$ represents this expansion, it is found that $\tilde{K}_{3}=K_{3}-\frac{1}{3}\left(* \varphi_{G}\right)^{3}$.
} 


$$
K_{q}(\gamma)=q^{\frac{3}{4}} \varphi_{G} J_{q}(\gamma)
$$

between the Kauffman bracket and Jones knot polynomials in the variable $q=e^{\Lambda}$ ( $\Lambda$ is the cosmological constant and in (69) the vertical framing is assumed).

\subsection{The Mandelstam identities}

As it is known, the Kauffman bracket is formally given by the expectation value of a Wilson loop in Chern-Simons theory [8]:

$$
K_{\Lambda}=<W(\gamma)>_{\Lambda}
$$

This basic relation provides a natural explanation of the fact that the knot invariants involved in the expansion of the Kauffman bracket in terms of the cosmological constant satisfy the Mandelstam identities in general. Under the light of (70), this property is inherited directly from the Wilson loops. In general, the verification of the Mandelstam identities for diffeomorphism invariant expressions of the general form (34) is quite nontrivial. In the context of quantum general relativity, the Mandelstam identities are basic requisites for the wavefunctions in the loop representation.

The Mandelstam identities for the members of a family can be checked systematically by means of equations (9)-(11). However, in the case of the family $\{\psi\}_{4}^{6}$ an immediate analysis follows from the result (67). In first place, all the members of a family are cyclic by construction. For the first two families $\{\psi\}_{2}^{2}$ and $\{\psi\}_{3}^{4}$, it is straightforward to see that the invariants $J_{2}$ and $\varphi_{G}$ satisfy the requirements (10) and (11). The $*$-product of an arbitrary number of Gauss invariants can be written in the following way: $\left(* \varphi_{G}\right)^{n}=\varphi_{G}^{n} \ldots X^{S[\cdots]}$, where $S[\cdots]$ is the average over the totally symmetric permutation of indices. This means that $\left(* \varphi_{G}\right)^{n}$ automatically satisfies the symmetry identities (10) and (11) for any $n$. On the other side, we know that $K_{3}=\tilde{K}_{3}+\frac{1}{3}\left(* \varphi_{G}\right)^{3}$ with $\tilde{K}_{3}$ the third coefficient of the expansion $K_{\Lambda}$ of the Kauffman bracket in the cosmological constant. From the general result (70) we conclude that the analytic expression $K_{3}$ would satisfy all the Mandelstam identities by construction. With respect to $J_{3}$, the result (68) shows that the behaviour of this invariant with respect to the second and third Mandelstam identities,

$$
\begin{aligned}
&\left(J_{3}\right) \boldsymbol{\mu} \stackrel{?}{=}\left(J_{3}\right) \overline{\boldsymbol{\mu}} \\
&\left(J_{3}\right)_{\boldsymbol{\alpha} \boldsymbol{\beta} \boldsymbol{\pi}}+\left(J_{3}\right)_{\boldsymbol{\alpha} \boldsymbol{\beta} \overline{\boldsymbol{\pi}}} \stackrel{?}{=}\left(J_{3}\right)_{\boldsymbol{\beta} \boldsymbol{\alpha} \boldsymbol{\pi}}+\left(J_{3}\right) \boldsymbol{\beta} \boldsymbol{\alpha} \overline{\boldsymbol{\pi}}
\end{aligned}
$$

essentially depends of the properties of the product $J_{2} * \varphi_{G}$ with respect to the same symmetry requirements. In general, the overline symmetry (71) is preserved under the $*$-product but the identity $(72)$ is not. This means that $J_{3}$ 
would inherit the property $(71)$ but not $(72)]^{13}$. The above discussion permits to conclude that:

1. All the wavefunctions $\psi_{1}, \cdots, \psi_{4}$ given by expressions (54)-(57) are invariant under the overline operation (71).

2. Only $\psi_{1}$ and $\psi_{2}$ are invariant under the property (72).

It is important to remark that for more complicated cases (i.e. for higher ranks families) the analysis of the Mandelstam identities could be worked in a systematic way.

\section{Conclusions and comments}

A systematic procedure to build up structured sets of diffeomorphism invariants has been developed. The method is based on the properties of the extended loop calculus.

A first point to remark is that the procedure of construction is generic in the sense that it does not respond to any single topological quantum field theory. In this respect, observe that the Chern-Simons propagators can be considered merely a support for the extended loop calculus and that a more basic mechanism (involving more general objects) could underlie the analysis. Indeed, it could be possible that a similar situation would occur with other building blocks (if exist) for the wavefunction's propagators.

The fact that there is not a unique topological quantum field theory of the Chern-Simons type underlying the procedure of construction has the following important consequence: the extended knot families would contain all the diffeomorphism invariant expressions characterized by the Chern-Simons propagators. This fact opens new possibilities to explore the analytic properties of knots. For the quantum gravity case we have the additional benefit to dispose of a systematic control of the Mandelstam symmetry requirements. The possibility of a systematic analysis of the Mandelstam's identities for diffeomorphism invariants is of relevance for the study of the Wheeler-DeWitt equation. Of particular interest is the possibility to generate at higher rank families invariants with the same analytic properties that the second coefficient $J_{2}$. The $J_{2}$ invariant is the only known (nontrivial) analytic expression that fulfills all the Mandelstam symmetry requirements and that is annihilated by the vacuum Hamiltonian constraint (the Brügmann-Gambini-Pullin solution). Also, it is a true diffeomorphism (ambient isotopic ) invariant not affected by framing ambiguities. In the context of the extended knot families, $J_{2}$ is the first analytic expression that appears after the most elementary Gauss invariant.

A more basic question is the problem to relate the individual solutions of the Hamiltonian constraint with some knot polynomial. As we have mention,

\footnotetext{
${ }^{13}$ For ordinary loops $J_{3}$ recovers the property (72) for some privileged topologies of the loop at the intersecting points. See [15] for a more detailed discussion.
} 
$J_{2}$ is related to both Alexander-Conway and Jones polynomials. The possibility that the expansion $J_{q}(\gamma)$ in the variable $q=e^{\Lambda}$ could be the general solution of the Wheeler-DeWitt equation without cosmological constant was suggested by Brügmann, Gambini and Pullin [23]. Their conjecture was mainly based on the formal relationship (69) existing between the Jones and the Kauffman bracket expansions. The analysis of this conjecture to third order in the cosmological constant has shown that the third coefficient of the Jones polynomial is not annihilated by the vacuum Hamiltonian constraint in the general case 157. On the other hand, the Alexander-Conway knot polynomial is associated with the expectation value of a Wilson type functional of a BF theory [24]. One can see that the topological quantity $<W(\gamma)>_{\mathrm{BF}}$ would satisfy a different sort of Mandelstam identities that the usual Wilson loop expectation value. For this reason, the knot invariants included in the perturbative expansion of $<W(\gamma)>_{\mathrm{BF}}$ could not be considered in principle good candidates

for quantum states of gravity. The above punctuations stress again the role of the $J_{2}$ invariant. Is this peculiar invariant a "strange" (unique) case or there exist "partners" that share its same properties? The problem of the existence of a knot polynomial associated with the space of states of quantum gravity remains open. It is possible that a systematic study of extended knot families could bring new insights to this question.

\section{Acknowledgments}

I want to thank R. Gambini and J. Pullin for many fruitful discussions. In special, I thank C. Di Bartolo by his comments and criticisms.

\section{Appendix}

In this appendix we shall include a list of useful results. We have:

$$
\begin{aligned}
& \mathcal{C}_{a x} C_{\mu_{1} \ldots \mu_{5}}^{1} X^{\mu_{1} \ldots \mu_{5}}=\left\{g_{\mu_{4}[a x} g_{b x] \mu_{3}} g_{\mu_{1} \mu_{2}}+g_{\mu_{4}[a x} g_{b x] \mu_{1}} g_{\mu_{2} \mu_{3}}\right. \\
& +g_{\mu_{2}[a x} g_{b x] \mu_{1}} g_{\mu_{3} \mu_{4}}+\epsilon_{a b c}\left(\phi_{x_{1}}^{c x}-\phi_{x_{4}}^{c x}\right) g_{\mu_{1} \mu_{4}} g_{\mu_{2} \mu_{3}}+\epsilon_{a b c}\left(\phi_{x_{1}}^{c x}-\phi_{x_{2}}^{c x}+\phi_{x_{3}}^{c x}\right. \\
& \left.\left.-\phi_{x_{4}}^{c x}\right) g_{\mu_{1} \mu_{2}} g_{\mu_{3} \mu_{4}}\right\} X^{\left(b x \mu_{1} \mu_{2} \mu_{3} \mu_{4}\right)_{c}}+\left\{2 g_{\mu_{1}[a x} h_{b x] \mu_{2} \mu_{3}}\right. \\
& \left.+\epsilon_{a b c}\left(\phi_{x_{3}}^{c x}-\phi_{x_{1}}^{c x}\right) h_{\mu_{1} \mu_{2} \mu_{3}}+2 \epsilon_{a b c} \phi_{z}^{c x}\left(\phi_{x_{3}}^{d z}-\phi_{x_{2}}^{d z}\right) g_{\mu_{1} d z} g_{\mu_{2} \mu_{3}}\right\} X^{\left(b x \mu_{1} \mu_{2} \mu_{3}\right)_{c}} \\
& \mathcal{C}_{a x} C_{\mu_{1} \ldots \mu_{5}}^{2} X^{\mu_{1} \ldots \mu_{5}}=\left\{-g_{\mu_{1}[a x} g_{b x] \mu_{3}} g_{\mu_{2} \mu_{4}}-g_{\mu_{2}[a x} g_{b x] \mu_{3}} g_{\mu_{1} \mu_{4}}\right. \\
& -g_{\mu_{2}[a x} g_{b x] \mu_{4}} g_{\mu_{1} \mu_{3}}+\epsilon_{a b c}\left(\phi_{x_{2}}^{c x}-\phi_{x_{3}}^{c x}\right) g_{\mu_{1} \mu_{4}} g_{\mu_{2} \mu_{3}}+\epsilon_{a b c}\left(\phi_{x_{1}}^{c x}+\phi_{x_{2}}^{c x}-\phi_{x_{3}}^{c x}\right. \\
& \left.\left.-\phi_{x_{4}}^{c x}\right) g_{\mu_{1} \mu_{3}} g_{\mu_{2} \mu_{4}}\right\} X^{\left(b x \mu_{1} \mu_{2} \mu_{3} \mu_{4}\right)_{c}}-\left\{2 g_{\mu_{1}[a x} h_{b x] \mu_{2} \mu_{3}}\right. \\
& \left.+\epsilon_{a b c}\left(\phi_{x_{3}}^{c x}-\phi_{x_{1}}^{c x}\right) h_{\mu_{1} \mu_{2} \mu_{3}}+2 \epsilon_{a b c} \phi_{z}^{c x}\left(\phi_{x_{3}}^{d z}-\phi_{x_{2}}^{d z}\right) g_{\mu_{1} d z} g_{\mu_{2} \mu_{3}}\right\} X^{\left(b x \mu_{1} \mu_{2} \mu_{3}\right)_{c}}
\end{aligned}
$$




$$
\begin{aligned}
& \mathcal{C}_{a x} C^{1}{ }_{\mu_{1} \ldots \mu_{6}} X^{\mu_{1} \ldots \mu_{6}}=\left\{g_{\mu_{2}[a x} g_{b x]} \mu_{3} g_{\mu_{1} \mu_{4}}\right. \\
& \left.+\epsilon_{a b c}\left(\phi_{x_{3}}^{c x}-\phi_{x_{2}}^{c x}\right) g_{\mu_{1} \mu_{3}} g_{\mu_{2} \mu_{4}}\right\} X^{\left(b x \mu_{1} \mu_{2} \mu_{3} \mu_{4}\right)_{c}} \\
& \mathcal{C}_{a x} C^{2}{ }_{\mu_{1} \ldots \mu_{6}} X^{\mu_{1} \ldots \mu_{6}}=\left\{g_{\mu_{4}[a x} g_{b x]} \mu_{1} g_{\mu_{2} \mu_{3}}\right. \\
& \left.+\epsilon_{a b c}\left(\phi_{x_{1}}^{c x}-\phi_{x_{4}}^{c x}\right) g_{\mu_{1} \mu_{2}} g_{\mu_{3} \mu_{4}}\right\} X^{\left(b x \mu_{1} \mu_{2} \mu_{3} \mu_{4}\right)_{c}} \\
& \mathcal{C}_{a x} C^{3}{ }_{\mu_{1} \ldots \mu_{6}} X^{\mu_{1} \ldots \mu_{6}}=\left\{g_{\mu_{2}[a x} g_{b x] \mu_{1}} g_{\mu_{3} \mu_{4}}+g_{\mu_{4}[a x} g_{b x] \mu_{3}} g_{\mu_{1} \mu_{2}}\right. \\
& +\epsilon_{a b c}\left(\phi_{x_{3}}^{c x}-\phi_{x_{2}}^{c x}\right) g_{\mu_{1} \mu_{2}} g_{\mu_{3} \mu_{4}} \\
& \left.+\epsilon_{a b c}\left(\phi_{x_{1}}^{c x}-\phi_{x_{4}}^{c x}\right) g_{\mu_{1} \mu_{4}} g_{\mu_{2} \mu_{3}}\right\} X^{\left(b x \mu_{1} \mu_{2} \mu_{3} \mu_{4}\right)_{c}} \\
& \mathcal{C}_{a x} C^{4}{ }_{\mu_{1} \ldots \mu_{6}} X^{\mu_{1} \ldots \mu_{6}}=\left\{g_{\mu_{1}[a x} g_{b x] \mu_{3}} g_{\mu_{2} \mu_{4}}+g_{\mu_{3}[a x} g_{b x] \mu_{2}} g_{\mu_{1} \mu_{4}}\right. \\
& +g_{\mu_{2}[a x} g_{b x] \mu_{4}} g_{\mu_{1} \mu_{3}}+\epsilon_{a b c}\left(\phi_{x_{3}}^{c x}-\phi_{x_{2}}^{c x}\right) g_{\mu_{1} \mu_{4}} g_{\mu_{2} \mu_{3}} \\
& \left.+\epsilon_{a b c}\left(\phi_{x_{2}}^{c x}-\phi_{x_{1}}^{c x}+\phi_{x_{4}}^{c x}-\phi_{x_{3}}^{c x}\right) g_{\mu_{1} \mu_{3}} g_{\mu_{2} \mu_{4}}\right\} X^{\left(b x \mu_{1} \mu_{2} \mu_{3} \mu_{4}\right)_{c}} \\
& \mathcal{C}_{a x} C^{5}{ }_{\mu_{1} \ldots \mu_{6}} X^{\mu_{1} \ldots \mu_{6}}=\left\{g_{\mu_{1}[a x} g_{b x] \mu_{4}} g_{\mu_{2} \mu_{3}}+g_{\mu_{1}[a x} g_{b x] \mu_{2}} g_{\mu_{3} \mu_{4}}\right. \\
& +g_{\mu_{3}[a x} g_{b x] \mu_{1}} g_{\mu_{2} \mu_{4}}+g_{\mu_{3}[a x} g_{b x] \mu_{4}} g_{\mu_{1} \mu_{2}}+g_{\mu_{4}[a x} g_{b x] \mu_{2}} g_{\mu_{1} \mu_{3}} \\
& +\epsilon_{a b c}\left(\phi_{x_{2}}^{c x}-\phi_{x_{1}}^{c x}+\phi_{x_{4}}^{c x}-\phi_{x_{3}}^{c x}\right)\left(g_{\mu_{1} \mu_{2}} g_{\mu_{3} \mu_{4}}+g_{\mu_{1} \mu_{4}} g_{\mu_{2} \mu_{3}}\right) \\
& \left.+\epsilon_{a b c}\left(\phi_{x_{1}}^{c x}-\phi_{x_{4}}^{c x}\right) g_{\mu_{1} \mu_{3}} g_{\mu_{2} \mu_{4}}\right\} X^{\left(b x \mu_{1} \mu_{2} \mu_{3} \mu_{4}\right)_{c}}
\end{aligned}
$$

\section{References}

[1] A. Ashtekar, Phys. Rev. Lett. 57 (1986) 2244; Phys. Rev. D36 (1987) 1587.

[2] C. Rovelli and L. Smolin, Phys. Rev. Lett. 61 (1988) 1155; Nucl. Phys. B331 (1990) 80.

[3] B. Brügmann, R. Gambini and J. Pullin, Phys. Rev. Lett. 68 (1991) 431.

[4] See for example L. Kauffman, "Knots and Physics", Series on Knots and Everything Vol. I, World Scientific (1991) and references therein.

[5] E. Witten, Comm. Math. Phys. 121 (1989) 351.

[6] E. Guadagnini, M. Martellini and M. Mintchev, Nucl. Phys. B227 (1989) 111. 
[7] A. Cattaneo, P. Cotta-Ramusino and M. Martellini, preprint IFUM 478/FT, hep-th/9407070.

[8] See for example R. Gambini and J. Pullin, "Loops, knots, gauge theories and quantum gravity", Cambridge University Press (to appear).

[9] B. Brügmann, R. Gambini and J. Pullin, Nucl. Phys. B385 (1992) 587.

[10] A. Ashtekar, C. Rovelli, L. Smolin, Phys. Rev. Lett. 69 (1992) 237.

[11] C. Di Bartolo, R. Gambini, J. Griego and J. Pullin, J. Math. Phys. 36 (1995) 6510.

[12] C. Di Bartolo, R. Gambini and J. Griego, Comm. Math. Phys. 158 (1993) 217.

[13] C. Di Bartolo, R. Gambini, J. Griego, J. Pullin, Phys. Rev. Lett. 72 (1994) 3638 .

[14] C. Di Bartolo, R. Gambini and J. Griego, Phys. Rev. D51 (1995) 502.

[15] J. Griego, IFFC preprint 95-05 (Montevideo), gr-qc/9510051.

[16] J. Griego, IFFC preprint 95-06 (Montevideo), gr-qc/9510050.

[17] J. Griego, "A renormalization of the Hamiltonian constraint of quantum gravity using extended loops", in preparation.

[18] J. Griego, IFFC preprint 95-09 (Montevideo), gr-qc/9512011 (to appear in Proceedings of the LASSF II Theoretical Physics, Ed. A. Restuccia et al., U.S.B. Caracas (1995)).

[19] R. Gambini and L. Leal, IFFI preprint 91-01 (Montevideo), unpublished.

[20] C. Di Bartolo, private communication.

[21] R. Gambini and A. Trias, Phys. Rev. D27 (1983) 2930.

[22] C. Di Bartolo and J. Griego, Phys. Lett. B317 (1993) 540.

[23] B. Brügmann, R. Gambini and J. Pullin, Gen. Rel. Grav. 25 (1993) 1.

[24] A. Cattaneo, P. Cotta-Ramusino, J. Fröhlich and M. Martellini, preprint IFUM 503/FT, hep-th/9505027. 